\title{
Three-dimensional Fermi surface determination by angle-resolved photoelectron spectroscopy
}

\author{
K. Rossnagel, L. Kipp, and M. Skibowski \\ Institut für Experimentelle und Angewandte Physik, Universität Kiel, D-24098 Kiel, Germany \\ C. Solterbeck, T. Strasser, and W. Schattke \\ Institut für Theoretische Physik und Astrophysik, Universität Kiel, D-24098 Kiel, Germany \\ D. Voß, P. Krüger, A. Mazur, and J. Pollmann \\ Institut für Festkörpertheorie, Universität Münster, D-48149 Münster, Germany
}

(Received 4 October 2000; published 7 March 2001)

\begin{abstract}
Angle-resolved photoelectron spectroscopy (ARPES) is commonly applied to map the shape of Fermi surfaces. Here we quantify the errors of simple criteria for extracting Fermi vectors by ARPES that are induced by strongly varying matrix elements. Sophisticated methods for determining the three-dimensional Fermi vector based on temperature and photon energy dependent photoemission are discussed with reference to data of the quasi-two-dimensional system $1 T$-TiTe 2 .
\end{abstract}

DOI: 10.1103/PhysRevB.63.125104

PACS number(s): 71.18.+y, 73.20.At, 79.60.-i

\section{INTRODUCTION}

The Fermi surface (FS) of a metal is intimately related to many of its low-energy properties such as transport, specific heat, and magnetic susceptibility. ${ }^{1}$ The canonical method for FS determination is based on the de Haas-van Alphen effect and measures extremal cross sections of the FS in a plane normal to an applied magnetic field. ${ }^{2}$ While this bulksensitive technique requires rather low temperatures and almost defect-free crystals, angle-resolved photoelectron spectroscopy (ARPES) has emerged as an alternative approach to FS determination which can overcome these restrictions. In particular, ARPES has played a key role in the study of the high-temperature superconductors (HTSC's) and other correlated materials. ${ }^{3}$

During the last few years, a method based on ARPES has been developed that provides a direct image of twodimensional FS cross sections. ${ }^{4,5}$ In this FS mapping technique, one records the angular (and hence $\mathbf{k}_{\|}$) distribution of only those photoelectrons that are excited from a small energy window centered on the Fermi energy. The Fermi level crossing of a conduction band, i.e., its Fermi vector, may then appear either as a maximum in the photoemission intensity or, in the case of narrow band systems, as a maximum in the momentum space gradient of the (energy-integrated) ARPES intensity. Formally, these interpretations of FS maps rely on the assumption that ARPES measures the oneparticle spectral function times the Fermi-Dirac function and matrix elements do not play a significant role. However, in the light of the lively discussion about the true topology and character of the normal state FS's of the HTSC's in general, and of $\mathrm{Bi}_{2} \mathrm{Sr}_{2} \mathrm{CaCu}_{2} \mathrm{O}_{8+\Delta}$ in particular, ${ }^{6-8}$ it turns out that the photon energy and wave vector dependence of the matrix elements, if not properly accounted for, can lead to strong misinterpretations of ARPES data.

Employing high-resolution photoelectron spectroscopy, we explicitly demonstrate and quantify here the effect of matrix elements on the different criteria for FS determination. Moreover, we present a recently developed, highly ac- curate method for determining the Fermi vector parallel to the surface. ${ }^{9}$ In this technique, intensity modifications due to the matrix elements are eliminated by examining the temperature variation of ARPES integrals. Finally, we show that the Fermi vector component perpendicular to the surface can be determined with moderate accuracy by comparing photoemission measurements at various photon energies with band structure calculations.

All results described in this paper were obtained from the quasi-two-dimensional Fermi liquid reference material $1 T$-TiTe 2 , which crystallizes in the $\mathrm{CdI}_{2}$ structure [space group $P \overline{3} M 1\left(D_{3 d}^{3}\right)$ ]: the hexagonal metal layers are sandwiched between anion sheets in such a way that each Ti atom is octahedrally coordinated to six Te atoms and each TeTi-Te sandwich is separated from the adjacent one by a van der Waals-type gap. Since $1 T-\mathrm{TiTe}_{2}$ does not show any indication of electronic or structural phase transitions, exhibits a high-quality surface, and has a Ti $3 d$-like conduction band that is well separated from other emissions, ${ }^{10-12}$ it may serve as an ideal candidate for assessing the accuracy of FS determination of quasi-two-dimensional systems by ARPES.

\section{EXPERIMENT}

Clean $1 T-\mathrm{TiTe}_{2}$ samples grown by chemical vapor transport were prepared by cleavage in ultrahigh vacuum. Photoemission spectra were taken with synchrotron radiation supplied from the beamlines W3.2 and BW3 of the storage ring DORIS III at Hamburg Synchrotron Radiation Laboratory (HASYLAB) using our angular spectrometer for photoelectrons with high-energy resolution (ASPHERE). ASPHERE is a $180^{\circ}$ spherical analyzer with a three-channeltron detector and is mounted on a two-axis goniometer with computercontrolled stepper motors, which enables an absolute angular precision of better than $0.1^{\circ}$. In the EDC (energy distribution curve) mode the energy and angular resolution were set to $30 \mathrm{meV}$ and $1^{\circ}$ full width at half maximum (FWHM), the photoelectron angular distributions were recorded with an overall energy resolution of $60 \mathrm{meV}$. The position of the 
Fermi level was determined from photoemission spectra of polycrystalline gold which was in electrical contact with the sample. ${ }^{13}$ All spectra shown were normalized to the incoming photon flux and no further data manipulation such as smoothing, symmetrization, or background correction was done.

\section{THEORY}

The band structure calculations were carried out employing density functional theory within the local-density approximation. ${ }^{14}$ We have used the norm-conserving, nonlocal pseudopotentials of Bachelet, Hamann, and Schlüter. ${ }^{15}$ The exchange-correlation energy was taken into account using the Ceperley-Alder ${ }^{16}$ form as parametrized by Perdew and Zunger. ${ }^{17}$

As a basis to represent the wave functions, we employ 130 Gaussian orbitals of $s, p, d$, and $s^{*}$ symmetry per unit cell and spin. These orbitals are localized at the atomic positions, namely, 50 at each $\mathrm{Ti}$ and 40 at each Te atom. The decay constants of the Gaussians are $\{0.17,0.47,1.30,3.56$, $9.95\}$ for $\mathrm{Ti}$ and $\{0.17,0.43,1.05,2.60\}$ for $\mathrm{Te}$ (in atomic units). A linear mesh of about $0.13 \AA$ in real space is used for the representation of the charge density and the potential.

The spin-orbit interaction is considered in each step of the iteration. It is treated in an on-site approximation, i.e., only integrals with the same location of the Gaussian orbitals and the spin-orbit potential are taken into account. The lattice parameters used in the calculations $\operatorname{are}^{18} a=3.777 \AA$, $c$ $=6.498 \AA$, and $z=0.2628 \mathrm{c}$.

\section{FERMI SURFACE MAPPING BY ARPES}

ARPES data on band dispersions naturally also contain information on the Fermi surface and its topology. This is illustrated in Fig. 1, showing photoemission spectra from layered $1 T-\mathrm{TiTe}_{2}$. The dispersion of bands in the EDC's measured along the $\Gamma M$ and $\Gamma K$ directions is visualized by an intensity map in the energy versus wave vector plane [Fig. 1(a)] and thus can be compared directly with the calculated band structure [Fig. 1(b)]. In addition to the good overall agreement of experiment and theory, one can easily identify the bands that generate the FS, i.e., the two strongly dispersive Te $5 p$ bands around the $\Gamma$ point and the narrow $\mathrm{Ti}$ $3 d$-like band at the $M$ point, which is well separated from other emissions. Along the $\Gamma M$ direction, the respective crossing points of the measured bands with the Fermi level are roughly located at $20 \%$ and $60 \%$ of the $\Gamma M$ distance $\left(0.96 \AA^{-1}\right)$.

Human subjectivity naturally plays an important role in identifying and tracing the dispersive quasiparticle peaks in such a data set. Nevertheless, there are a number of simple criteria that are commonly applied to extract Fermi vectors from a series of EDC's. First, the energy position of the midpoint of the leading edge of the EDC may be observed, with the Fermi vector found at the point where this quantity reaches its maximum value. Second, the Fermi level crossing may be ascribed to the point where the binding energy of the peak maximum or the peak width has its minimum value. (a) Experiment

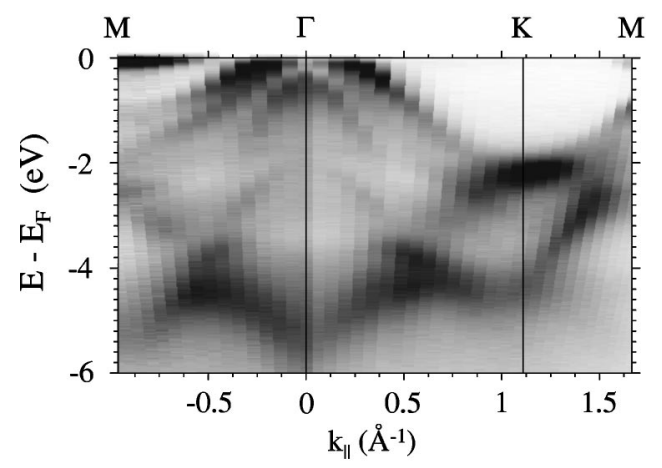

(b) Theory

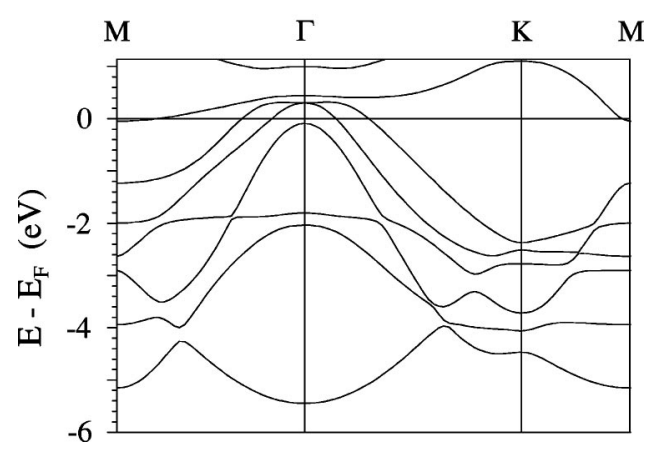

FIG. 1. (a) Experimental valence bands for $1 T-\mathrm{TiTe}_{2}$ obtained by ARPES in the EDC mode at room temperature with $h \nu$ $=24 \mathrm{eV}$. Photoemission intensity values are represented in a linear gray scale with black corresponding to high intensity. (b) Corresponding calculated band structure.

Third, the center of gravity of the quasiparticle peak can be traced along the selected path in $\mathbf{k}$ space and extrapolated to the intersection with the Fermi energy. Although these guidelines are adequate for gaining a rough qualitative overview of FS topology, they cannot provide an accurate and reliable means of extracting Fermi vectors because they do not rely on a solid theoretical basis. In particular, they are usually not implemented with proper consideration of the Fermi-Dirac cutoff function, the experimental resolutions, and the matrix elements of the photoemission process.

Recently, an alternative technique of Fermi surface mapping has been introduced, in which one records the angular distribution of only those photoelectrons that are excited from a narrow energy window centered on the Fermi energy. ${ }^{4,5}$ By acquiring a single data point rather than a complete EDC for each $\mathbf{k}_{\|}$vector, it is possible to densely sample the entire Brillouin zone (BZ) much faster and more completely than is feasible in the conventional EDC mode, even though one naturally loses information on band dispersion and Fermi velocity. The essential assumption that is made in this technique is that the Fermi level crossing of a conduction band will appear as a local maximum in the detected photoemission intensity ("maximum intensity method").

Figure 2 shows FS maps of $1 T-\mathrm{TiTe}_{2}$ taken with two different photon energies $(h \nu=24 \mathrm{eV}$, top panel, and $h \nu$ $=98.4 \mathrm{eV}$, bottom panel). One can easily identify the two types of FS sheets that have already appeared in Fig. 1: the 
(a) $\mathrm{hv}=24 \mathrm{eV}$

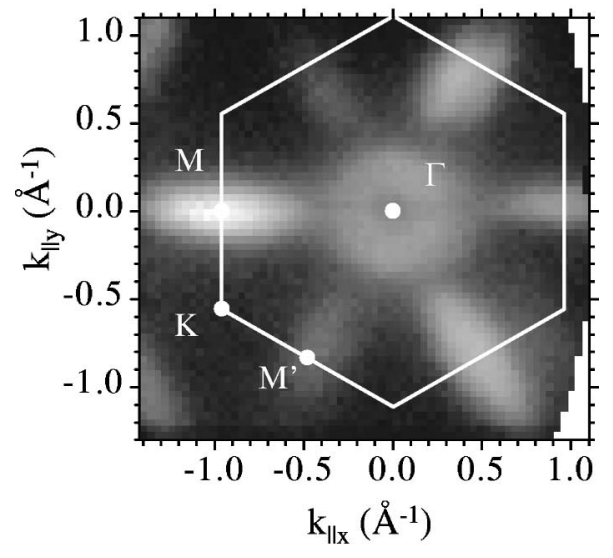

(b) Theory

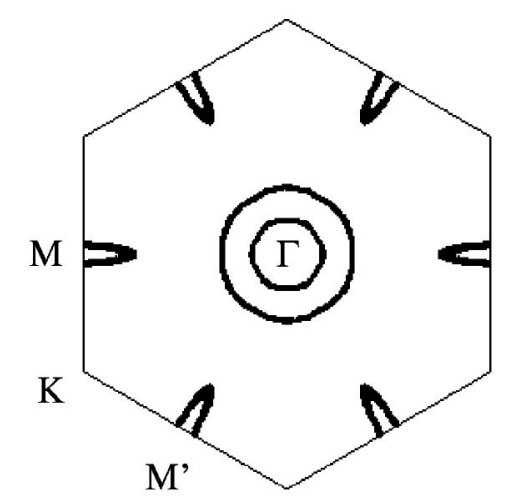

(c) $\mathrm{h} v=98.4 \mathrm{eV}$

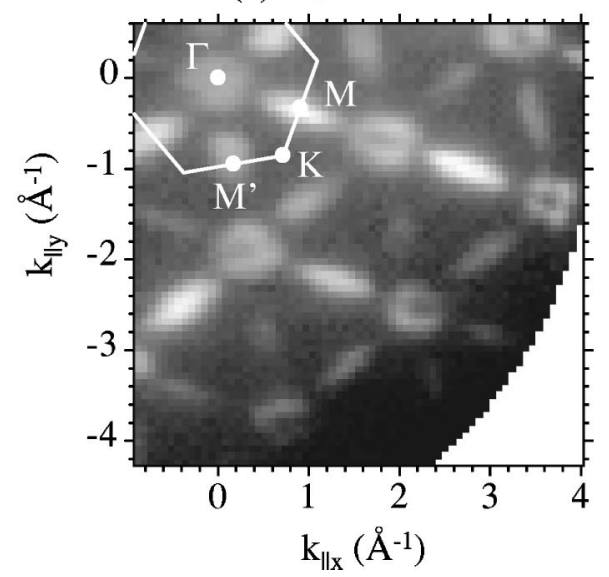

FIG. 2. Fermi surface mapping for $1 T-\mathrm{TiTe}_{2}$ at room temperature using photon energies of (a) $24 \mathrm{eV}$ and (c) $98.4 \mathrm{eV}$ compared to (b) theoretical Fermi surface cut at $k_{\perp}=0$ obtained by bulk band structure calculation. Photoemission intensity is represented in a logarithmic gray scale with white corresponding to high intensity. The Brillouin zone and the high-symmetry points are indicated.

small hole pocket at the center of the $\mathrm{BZ}$ derived from the Te $5 p$-like bands and the Ti $3 d$-related ellipsoidal electron pockets centered on the $M$ and $M^{\prime}$ points. ${ }^{19}$ In the photoelectron angular distribution with $h \nu=98.4 \mathrm{eV}$, this FS pattern is nicely repeated in higher BZ's. Note, however, that in both
FS maps, matrix element effects lead to strong variation of the photoemission intensity in equivalent parts of the BZ, particularly seen in the pronounced difference between $M$ and $M^{\prime}$ points. This corroborates the fact that, although the maximum intensity method has intuitive appeal and reproduces the FS topology quite well as compared to the theoretical Fermi surface cut in Fig. 2(b), the precise physical meaning of the quantity being measured cannot be interpreted in simple terms.

A third technique, which has been proposed very recently, can be justified from a much more physical point of view. ${ }^{20-22}$ Assuming the validity of the sudden approximation and neglecting photoelectron and experimental broadening, ARPES measures the product of the transition matrix element $I_{0}(\mathbf{k})$ with the one-particle spectral function $A(\mathbf{k}, \omega)$, i.e., the photocurrent is approximately given by $I(\mathbf{k}, \omega)=I_{0}(\mathbf{k}) A(\mathbf{k}, \omega) f(\omega) \quad$ ("spectral function interpretation"), ${ }^{23}$ where $f$ is the Fermi-Dirac function. If $I_{0}$ does not have any significant $\omega$ dependence, the energyintegrated ARPES spectrum will be proportional to the momentum distribution function $n(\mathbf{k})$ $=\int_{-\infty}^{\infty} d \omega f(\omega) A(\mathbf{k}, \omega),{ }^{24}$ modulated, of course, by the $\mathbf{k}$ dependence of the matrix element prefactor.

It is well known that, within the Fermi liquid framework, the momentum distribution function drops discontinuously at the FS, with the size of the step being directly related to the mass renormalization. ${ }^{25}$ Although at nonzero temperature and with finite experimental resolution, the discontinuity is smeared out, the measured $n(\mathbf{k})$ will still display rapid variations at the Fermi vectors. Under the assumption of weakly varying matrix elements, the location of the Fermi vectors can thus be determined from the extrema of the momentum space gradient of the energy-integrated ARPES intensity $\left|\boldsymbol{\nabla}_{\mathbf{k}}\left[I_{0}(\mathbf{k}) n(\mathbf{k})\right]\right| \approx\left|\boldsymbol{\nabla}_{\mathbf{k}} n(\mathbf{k})\right| \quad$ ("maximum gradient method"). Since the momentum distribution sum rule holds for many-body systems independent of the details of interaction, this approach to FS determination is also valid for strongly correlated and non-Fermi-liquid materials, if there is still an anomaly in $n(\mathbf{k})$ at $\mathbf{k}_{F}$.

In the experimental implementation of the maximum gradient method, photoemission intensity has to be accumulated over a sufficiently wide energy range, ideally the entire conduction band width. In practice, the energy integration is often replaced by the finite energy resolution of the photoelectron spectrometer, but there is nevertheless a need to check on the adequate energy integration window, especially in the case of overlapping bands, which may lead to spurious peaks in the momentum space gradient. For this reason, it is essential to retain energy distribution information, even if one is only interested in FS topology.

\section{THE EFFECT OF MATRIX ELEMENTS ON FERMI SURFACE MAPPING}

As noted in the previous section, the maximum intensity and the maximum gradient method are both affected by the $\mathbf{k}$ dependence of the matrix element factor, which may lead to uncertainties in the determination of Fermi vectors. In order to quantify this matrix element effect, we have determined 

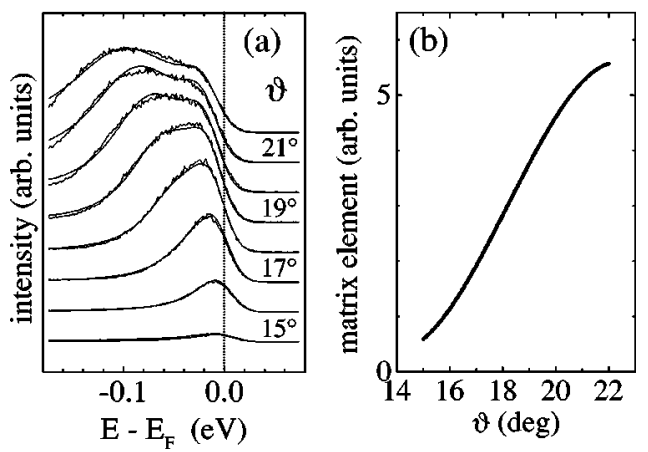

FIG. 3. (a) ARPES spectra of the Ti $3 d$-related band of $1 T-\mathrm{TiTe}_{2}$ along the $\Gamma M$ direction taken at $30 \mathrm{~K}$ with $h \nu$ $=19.5 \mathrm{eV}$ compared to line shape fit using a Fermi-liquid-type model. (b) $\mathbf{k}$ dependence of the matrix element factor as obtained from the fit.

the prefactor $I_{0}(\mathbf{k})$ and the band dispersion $\epsilon_{\mathbf{k}}$ close to $E_{F}$ for the model system $1 T$-TiTe 2 by analyzing the ARPES line shapes of the Ti $3 d$-like emission in terms of a refined Fermi liquid (FL) model. ${ }^{26-28}$ It was shown before that ARPES data of $1 T-\mathrm{TiTe}_{2}$ can be successfully described within a FL scenario. ${ }^{10,11,29}$ Here we want to focus on the Fermi level crossing and matrix elements.

Figures 3(a) and 4(a) show ARPES spectra of the Ti $3 d$-derived band along the $\Gamma M$ direction taken at low temperature $(30 \mathrm{~K})$ and a photon energy of $19.5 \mathrm{eV}$. At these experimental parameters, thermal and photoelectron broadening of the spectra are expected to be irrelevant. ${ }^{30}$ Note that the spectra are virtually free from any background and other emissions, except for a small Te $5 p$-related structure at $\sim 300 \mathrm{meV}$ binding energy and low emission angles [see Fig. 4(a)]. To fit the theoretical line shapes to the experimental data, the real parameters $I_{0}, \epsilon_{\mathrm{k}}$, and $Z$ and the complex $\Omega_{b}$ were used, ${ }^{31}$ where the latter two correspond to the quasiparticle weight and the background pole, respectively (see Refs. 28 and 29 for details). The finite angular and energy resolution of the spectrometer were taken into account via Gaussian distributions in momentum and energy space and, as an entire EDC series served as input vector, the $\mathbf{k}$ dependence of the fit parameters was modeled by smooth loworder polynomials. The results of this analysis are displayed in Fig. 3.

The complete model function is compared with the experimental data in Fig. 3(a). Not surprisingly, the FL-type model produces a good least squares fit of the spectra close to $\mathbf{k}_{F}$ with $Z$ and $\operatorname{Im}\left(\Omega_{b}\right)$ values similar to the ones found before $\left[Z=0.11-0.41, \operatorname{Im}\left(\Omega_{b}\right)=33-67 \mathrm{meV}\right]$. An important point is that the fit quality could be significantly improved by including the additional parameter $\operatorname{Re}\left(\Omega_{b}\right)=-39-19 \mathrm{meV}$, which means absence of particle-hole symmetry globally. However, at the Fermi vector, which is located at $15.5^{\circ}$ $\pm 0.1^{\circ}\left[k_{F \|}=(0.529 \pm 0.003) \AA^{-1}\right]$, the fitting procedure reveals $\operatorname{Re}\left(\Omega_{b}\right) \approx 0$, and thus $A\left(\mathbf{k}_{F},-\omega\right)=A\left(\mathbf{k}_{F}, \omega\right)$. Note that the fit quality becomes worse at higher emission angles, indicating that the FL model is valid only in a small energy and wave vector interval around $\left(E_{F}, \mathbf{k}_{F}\right)$.

In Fig. 3(b) the matrix element factor as obtained from the
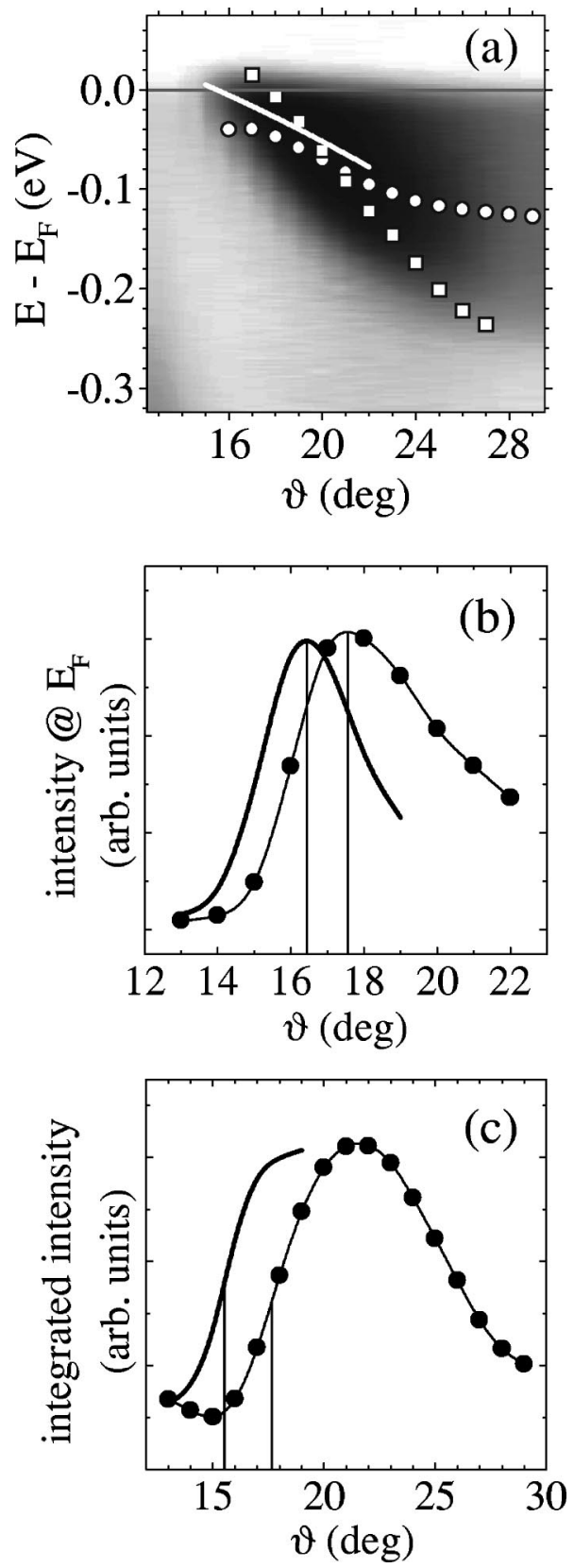

FIG. 4. (a) ARPES spectra of the Ti $3 d$-related band of $1 T-\mathrm{TiTe}_{2}$ along the $\Gamma M$ direction taken at $30 \mathrm{~K}$ with $h \nu$ $=19.5 \mathrm{eV}$. Photoemission intensity is represented in a linear gray scale with black corresponding to high intensity. The energy dispersion obtained by line shape analysis is indicated by the solid white line. The centers of gravity of the EDC's and the positions of the maxima of the MDC's are denoted by open circles and squares, respectively. Photoemission intensities (filled circles) (b) at the Fermi level and (c) integrated over the whole spectrum. Simulated intensities, using a Fermi-liquid-type spectral function with parameters obtained by line shape analysis and assuming a constant matrix element factor, are indicated by thick solid lines.

line shape analysis is shown. Obviously, the fitted $I_{0}(\mathbf{k})$ varies strongly with $\mathbf{k}$ giving a dominant contribution to the $\mathbf{k}$ dependence of the photoemission intensity on a relatively small $\mathbf{k}$ interval around $\mathbf{k}_{F}$. The fitted energy dispersion is 
shown in Fig. 4(a) (white line) together with the centers of gravity of the experimental EDC's (circles) and the positions of the peak maxima (squares) of the momentum (angular) distribution curves (MDC's), the latter being cuts through the data set at constant energy, the former at constant angle. While the center of gravity of the EDC's at least resembles the fitted excitation energy $\epsilon_{\mathrm{k}}$ for higher emission angles $\left(>18^{\circ}\right)$, the maxima of the MDC's are far off the fitted dispersion. In fact, the MDC's should be affected to much greater extent by the matrix elements than the EDC's, because the $\mathbf{k}$ variation of the matrix element factor [as shown in Fig. 3(b)] is supposed to be much stronger than its energy dependence, which may presumably be neglected in the small energy window $(\sim 300 \mathrm{meV})$ around $E_{F}$. Nevertheless, even the center of gravity of the EDC's cannot exactly reproduce the dispersion close to $\mathbf{k}_{F}$ because of the FermiDirac cutoff and possible extrapolations of the data points may lead to an error of up to $1.2^{\circ}\left(\Delta k=0.04 \AA^{-1}\right)$ in FS determination.

After this rather cumbersome determination of the Fermi vector involving an enormous computational effort, we now turn to the question as to what extent the factor $I_{0}(\mathbf{k})$ affects the more applicable maximum intensity and maximum gradient methods. In Figs. 4(b) and 4(c), we show the intensities at the Fermi level and the intensities integrated over the whole spectrum as taken from the data set of Fig. 4(a). The strong influence of the matrix element can directly be seen in the rapid decrease of the integrated intensity at high emission angles $\left(>23^{\circ}\right)$. Both criteria, the maximum intensity at $E_{F}$ as well as the maximum gradient of the energy-integrated intensity, locate the Fermi level crossing at $17.6^{\circ}\left(k_{\|}\right.$ $\left.=0.598 \AA^{-1}\right)$. With respect to the fitted value $\left(15.5^{\circ}\right)$, this corresponds to an experimental error in FS determination of $\Delta k=0.069 \AA^{-1}$ or $7 \%$ of the relevant $\mathrm{BZ}$ dimension induced by the $\mathbf{k}$ dependence of the matrix element. To further illustrate this strong effect, we have simulated photoemission intensities by convoluting the $\mathbf{k}$ dependent spectral function as obtained from the line shape analysis with the experimental resolutions under the assumption of a constant matrix element factor. While the point of inflection of the integrated intensity now coincides with the Fermi vector determined by the fitting procedure [see Fig. 4(c)], the position of the maximum of the simulated intensity at $E_{F}$ still deviates from this value by $0.9^{\circ}$ [Fig. 4(b)]. This explicitly demonstrates the fact that for narrow band systems at finite temperature and resolution, the maximum intensity method cannot produce reliable Fermi vectors even for negligible matrix elements.

\section{THE $\triangle T$ METHOD}

The essential point of the last section was that simple interpretations of ARPES intensities neglecting the influence of matrix elements may lead to substantial errors of FS determination. We will now show how Fermi vectors can be obtained very accurately from ARPES integrals that are taken at different temperatures. Assuming the spectral function interpretation $I(\mathbf{k}, \omega)=I_{0}(\mathbf{k}) A(\mathbf{k}, \omega) f(\omega)$, with $I_{0}$ being independent of $\omega$ and $T$, and provided $A\left(\mathbf{k}_{F},-\omega\right)$ $=A\left(\mathbf{k}_{F}, \omega\right)$ for all $\omega$ ("particle-hole symmetry"), it was shown before that $n\left(\mathbf{k}_{F}\right)$ is independent of temperature, ${ }^{24}$ while $n(\mathbf{k})$ for $\mathbf{k}$ near $\mathbf{k}_{F}$ is generally not. Accordingly, points $\mathbf{k}_{F}$ on the FS can be identified by a change of sign in the temperature variation of $n(\mathbf{k})$, i.e., the photoemission intensity integrated over the entire conduction band width. Here we want to compare this approach with the more accurate " $\Delta T$ method" which is based on the temperature dependence of ARPES intensities that are integrated over only a tiny energy interval centered on $E_{F} \cdot{ }^{9}$ The $\Delta T$ method has the advantage that all assumptions made are required to hold only in this very small energy window.

The difference of ARPES intensities taken at $E_{F}=0$ for temperatures $T_{1}$ and $T_{2}$ is given by

$$
\Delta I(\mathbf{k})=I_{0}(\mathbf{k}) \int_{-\varepsilon}^{\varepsilon} d \omega A(\mathbf{k}, \omega)\left[f\left(\omega, T_{1}\right)-f\left(\omega, T_{2}\right)\right] w(\omega),
$$

where $w$ is a symmetric energy resolution function which is zero outside $[-\varepsilon, \varepsilon]$. With respect to $\omega$, the difference of the Fermi-Dirac functions is odd, $A\left(\mathbf{k}_{F}, \omega\right)$ is even, and therefore the integral of their product over a symmetric energy window $^{32}$ vanishes. Thus, $\mathbf{k}=\mathbf{k}_{F}$ follows from $\Delta I(\mathbf{k})=0$. This is valid for all $A$ that are temperature independent for the intervals of interest and satisfy in $[-\varepsilon, \varepsilon]$ the requirements that $A\left(\mathbf{k}_{F},-\omega\right)=A\left(\mathbf{k}_{F}, \omega\right)$ and $A(\mathbf{k},-\omega) \neq A(\mathbf{k}, \omega)$ for $\mathbf{k} \neq \mathbf{k}_{F}$ near $\mathbf{k}_{F}$. A variety of spectral functions fulfill these conditions, including the Luttinger model,,$^{33}$ a suggestion by Matho, ${ }^{26,27}$ and two-dimensional ${ }^{34}$ and marginal Fermi liquids. ${ }^{35}$ Recently, the applicability of the criterion has been extended to arbitrary FL scenarios with quadratic damping. ${ }^{36}$ With high $\mathbf{k}$ resolution, the criterion generally remains stable.

In Fig. 5(a) we show photoemission spectra of $1 T-\mathrm{TiTe}_{2}$ along the $\Gamma M$ direction of the $\mathrm{BZ}$ for $30 \mathrm{~K}$ and $100 \mathrm{~K}$. The broadening of the Fermi-Dirac function due to temperature is evident. An important point in comparing data at different temperatures is the normalization of the EDC's. All spectra shown were normalized to the incoming photon flux and no further correction needed to be made: since layered $1 T-\mathrm{TiTe}_{2}$ exhibits a large nonreactive sample surface, the measurements do not suffer from adsorption of gases or the small thermal expansion of the sample holder. Intensities at the Fermi level measured with an energy resolution of 30 $\mathrm{meV}$ and intensities integrated from $-200 \mathrm{meV}$ to $50 \mathrm{meV}$ corresponding to the bandwidth are depicted in Figs. 5(b) and 5(c). The corresponding intensity differences are shown in the bottom panels.

According to the $\Delta T$ method, the Fermi vector is given by the intersection of the two curves where $\Delta I=0$ [Fig. 5(b)]. This point can be identified at $15.2^{\circ} \pm 0.1^{\circ}\left[k_{\|}=(0.519\right.$ $\left.\pm 0.003) \AA^{-1}\right]$ which agrees with the value obtained from the line shape analysis within an uncertainty of less than $2 \%$. The small deviation might be due to finite $\mathbf{k}$ resolution, which could be concluded from the fact that the experimental $\mathbf{k}$ resolution window does not lie within a regime of perfect point symmetry of $\Delta I(\mathbf{k})$ around $\Delta I=0$ [see Fig. 5(b), bottom panel]. Note that for a tiny energy interval the required fixed-k mode of photoemission corresponds to the fixedangle mode employed. As can be deduced from the symme- 

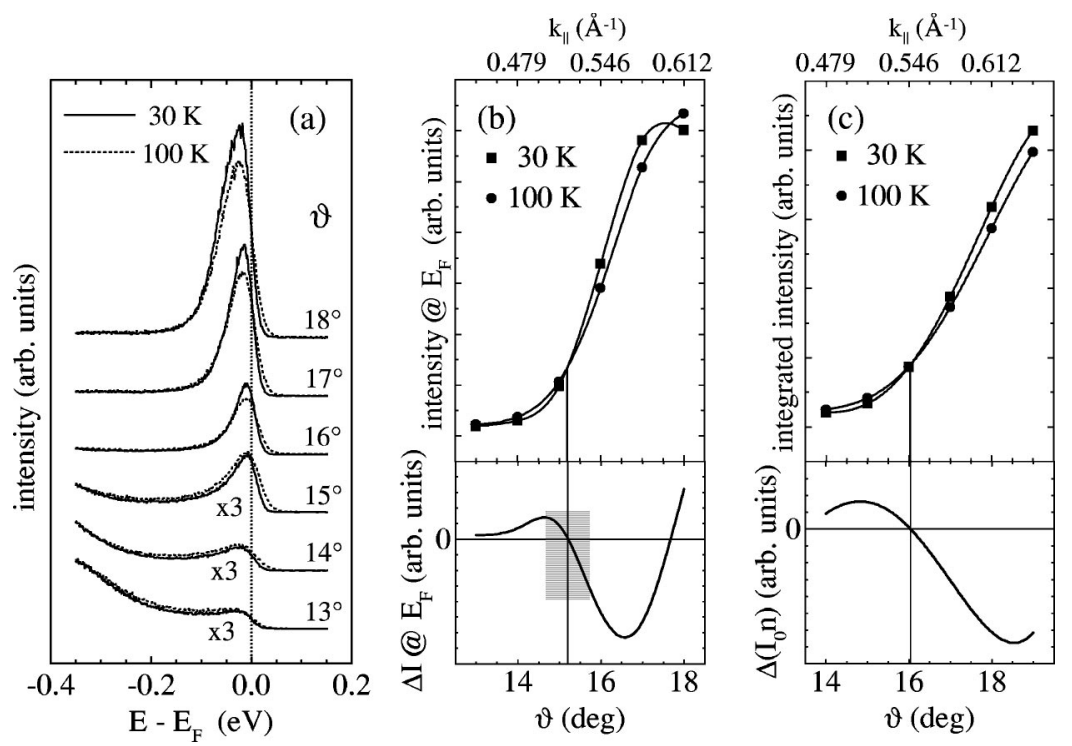

FIG. 5. (a) ARPES spectra of the $\mathrm{Ti}$ $3 d$-related band of $1 T-\mathrm{TiTe}_{2}$ along the $\Gamma M$ direction taken at $30 \mathrm{~K}$ and $100 \mathrm{~K}$ with $h \nu$ $=19.5 \mathrm{eV}$. (b) Intensities at the Fermi level and (c) intensities integrated from -200 to $50 \mathrm{meV}$. The intensity differences are shown in the bottom panels. The point of intersection gives the Fermi vector $(\Delta I=0)$. The hatched area corresponds to the experimental angular resolution window.

trized spectra at the Fermi vector $I\left(\mathbf{k}_{F}, \omega\right)+I\left(\mathbf{k}_{F},-\omega\right)$ $=I_{0}\left(\mathbf{k}_{F}\right) A\left(\mathbf{k}_{F}, \omega\right),{ }^{37}$ shown in Fig. 6(a), a possible influence due to temperature dependent broadening of $I_{0}\left(\mathbf{k}_{F}\right) A\left(\mathbf{k}_{F}, \omega\right)$ can be neglected in the experimental energy resolution window centered on the Fermi energy. In addition, the centers of gravity of the EDC's along the $\Gamma M$ direction taken at $30 \mathrm{~K}$ and $100 \mathrm{~K}$ indicate that mainly the width of the Ti $3 d$ band changes due to temperature, while the Fermi level crossing remains unaltered within an uncertainty of $0.2^{\circ}$ [see Fig. 6(b)].

For comparison, the point of temperature independent intensity integrated over the entire bandwidth can be found at $16.1^{\circ}$ [see Fig. 5(c)]. The larger deviation from the fitted value might result from a slight $T$ dependence of the overlapping Te $5 p$-related structure at low emission angles, which contributes to the integrated intensity but not to the intensity at $E_{F}$. Concerning the accuracy of this method, it is important to notice that all assumptions leading to $T$ independence of $n\left(\mathbf{k}_{F}\right)$ must hold in a much wider energy interval than is required for the $\Delta T$ method, the latter being thus more accurate and having wider application. However, both approaches to FS determination are at any rate superior to simple interpretations of ARPES intensities because they explicitly consider the $\mathbf{k}$ dependence of the transition matrix element.

\section{DETERMINATION OF $\mathbf{k}_{F \perp}$}

So far, we have discussed only the problem of determining the surface-parallel component of the Fermi vector $\mathbf{k}_{F \|}$, whereas a precise determination of the complete threedimensional Fermi vector additionally requires control of the surface-perpendicular component $k_{F \perp}$. Unfortunately, in an ARPES experiment one directly controls only the energy and surface-parallel momentum of the detected photoelectrons, while the surface-perpendicular momentum corresponding to an observed ARPES peak is implicitly given by the condition that the photon energy connects initial and final states in a direct transition. Thus, the exact determination of $k_{\perp}$ requires knowledge of the final state band dispersion, which is generally not available. However, for FS measurements of $\mathrm{Cu}$ and $1 T$-TiTe 2 it has been shown that the final states can reasonably well be approximated by a free-electron-like dispersion with a suitable inner potential $V_{0} \cdot{ }^{21,38}$

One way to map the shape of a three-dimensional FS by ARPES is to measure the photoemission intensity $I\left(\mathbf{k}_{\|}, E_{F}\right)$ as a function of the photon energy $h \nu$, i.e., one performs constant initial energy spectroscopy (CIS) using electrons from the Fermi level for an appropriate range of polar emission angles and photon energies that span the bulk BZ cross section. ${ }^{39}$ In Fig. 7(a) we show such a measurement for the $\Gamma M A L$ plane of $1 T-\mathrm{TiTe}_{2}$. The surface-parallel component of the wave vector was determined using the usual equation $k_{\|}=\sqrt{\left(2 m / \hbar^{2}\right) E_{k i n}} \sin \vartheta$ and $k_{\perp}$ was calculated from the formula $\left(E_{k i n}+V_{0}\right)=\left(\hbar^{2} / 2 m\right) \mathbf{k}^{2}$ assuming a free-electron final state band. The best fit between experiment and theory was obtained when an inner potential $V_{0}=14.0 \mathrm{eV}$ was used, which agrees well with the value found before $(14.5 \mathrm{eV}$ in Ref. 12). The topology of the theoretical FS cut (thick solid lines), with the Te $5 p$-related bands close to $\Gamma A$ and the Ti $3 d$-derived emission near $M L$, is clearly mimicked by the photoemission intensity map. However, note that the maxi-
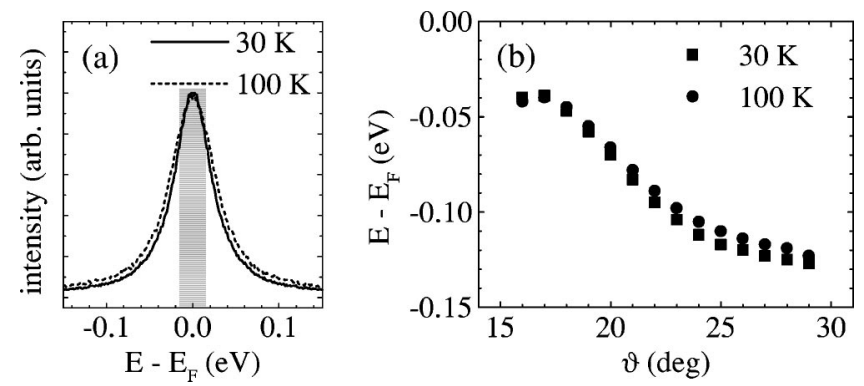

FIG. 6. (a) Symmetrized spectra of the Ti $3 d$ emission of $1 T-\mathrm{TiTe}_{2}$ at the Fermi vector taken at $30 \mathrm{~K}$ and $100 \mathrm{~K}$ with $h \nu$ $=19.5 \mathrm{eV}$. The hatched area corresponds to the experimental energy resolution window. (b) Centers of gravity of EDC's along the $\Gamma M$ direction for $30 \mathrm{~K}$ and $100 \mathrm{~K}$. 

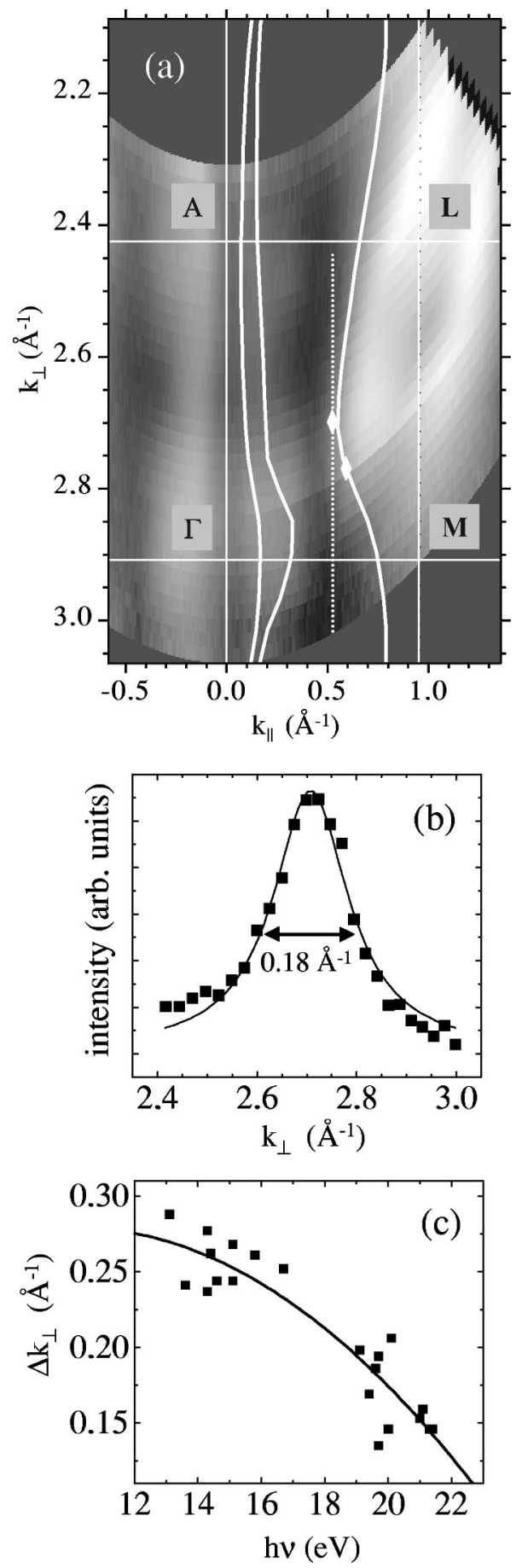

FIG. 7. (a) Fermi surface map of $1 T$-TiTe 2 obtained by ARPES at room temperature by employing polar angle scans at photon energies from 11 to $26 \mathrm{eV}$ compared to theoretical Fermi surface cut. The two diamonds mark Fermi level crossing points determined by the $\Delta T$ method. $k_{\perp}$ values are calculated under the assumption of a free-electron final state. Photoemission intensity is represented in a logarithmic gray scale with white corresponding to high intensity. The high-symmetry points of the Brillouin zone are indicated. The thin dotted line marks the vertical cut shown in (b) together with a Lorentzian fit. In (b) the intensity scale is linear. (c) Experimental energy dependent momentum broadening. mum photoemission intensity never coincides with the calculated FS, while on the other hand the $\Delta T$ method produces almost exact Fermi vectors (indicated by filled diamonds). ${ }^{40}$

Within this analysis, the surface-perpendicular component of the three-dimensional Fermi vector, whose component parallel to the surface has been determined at $h \nu=19.5 \mathrm{eV}$ by using the $\Delta T$ method, is $k_{F \perp}=2.698 \AA^{-1}$. If the inner potential was in error by as much as $2 \mathrm{eV}$, this would give an experimental error of $\Delta k_{\perp} \approx 0.1 \AA^{-1}$. However, the accuracy of the technique is essentially limited by the photoemission process itself. Since photoemission is a many-body phenomenon involving quasiparticles with finite lifetimes, the initial and final state wave functions in the solid are damped, which leads in particular to a relaxation of $k_{\perp}$ conservation. ${ }^{41}$ For instance, the eigenstates of photoelectrons from $\mathrm{Cu}$ are smeared out in $\mathbf{k}$ space by $\Delta k_{\perp} \approx 0.1 \AA^{-1}$ for $h \nu \approx 20 \mathrm{eV}{ }^{42}$

In Fig. 7(b) we show a vertical cut through the intensity map of Fig. 7(a). The Lorentzian function of width $\Delta k_{\perp}$ $=0.18 \AA^{-1}$, which has been fitted to the experimental intensities, approximates the distribution of the final state. This simple interpretation seems very likely since the photon energy dependent factors such as escape probability and matrix elements may be assumed to vary slowly with $h \nu$ and the initial state lifetime contribution is very small because the initial state lies at $E_{F} \cdot{ }^{41}$ The intrinsic $\mathbf{k}$-space error appears then to be $\Delta k_{\perp}=0.18 \AA^{-1}$. In fact, this value is an upper limit for final state broadening because the linewidth in a CIS spectrum with $k_{\|}=$const is approximately given by $\Gamma_{m}$ $=\left(v_{f \perp} / v_{i \perp}\right) \Gamma_{i}+\Gamma_{f},{ }^{43}$ where $\Gamma$ and $\hbar v_{\perp}=\partial E / \partial k_{\perp}$ denote the lifetime width and group velocity of the initial and final state, respectively, and $v_{i \perp}$ is very small for the present case.

Thus, the error in determining $k_{\perp}$ using the assumption of a free-electron-like final state is comparable to the intrinsic $\mathbf{k}$-space error for the energy range studied. Improvements to the accuracy of the technique should then arise not only from more sophisticated final state wave functions but also from different experimental conditions. One promising way is to use either very low $(<10 \mathrm{eV})$ or high $(>100 \mathrm{eV})$ photon energies $^{39}$ at which the mean free path of photoelectrons increases, giving smaller errors of $\Delta k_{\perp}<0.1 \AA^{-1} .42$ In Fig. 7 (c) we show the energy dependent momentum broadening for $1 T-\mathrm{TiTe}_{2}$ as obtained by vertical cuts through the intensity map in Fig. 7(a). While it remains an open question whether the measurement follows the universal curve ${ }^{44}$ for momentum broadening, i.e., the inverse electron mean free path, with the strong decrease of $\Delta k_{\perp}$ at low photon energies $(<10 \mathrm{eV})$, it is obvious that $\Delta k_{\perp}$ decreases for higher photon energies in accordance with the universal curve. Consequently, at photon energies higher than $100 \mathrm{eV}$ the intrinsic k-space error should be smaller than $0.05 \AA^{-1}$. On the other hand, one has to keep in mind that at $h \nu=100 \mathrm{eV}$, the wave vector of the photon, usually neglected, already has a value of $0.05 \AA^{-1}$, which again limits the accuracy of $\mathbf{k}$ determination due to the constraints of the photoemission process.

\section{CONCLUSIONS}

Within a spectral function interpretation of ARPES, we have discussed several criteria for determining surface- 
parallel Fermi vectors with special reference to the question of how much they are influenced by the $\mathbf{k}_{\|}$dependence of the transition matrix element. Employing high-resolution ARPES on the layered compound $1 T$ - $\mathrm{TiTe}_{2}$, we have explicitly shown that simple interpretations of photoemission intensities neglecting the matrix element factor may lead to large errors in the extracted Fermi vector. A method for quantitative Fermi surface determination based on the temperature variation of ARPES integrals taken at the Fermi level is further elucidated and is demonstrated to produce highly accurate results, aiming at an experimental error of less than $1 \%$ of typical Brillouin zone dimensions even for strongly varying matrix elements. By comparing photon en- ergy dependent measurements with a band structure calculation, we have finally determined the surface-perpendicular Fermi vector component with an accuracy of $0.1-0.2 \AA^{-1}$ and discussed the limitations due to the constraints of the photoemission process. In conclusion, the methods described in this paper offer reliable and precise ways to experimentally study three-dimensional Fermi surfaces and interrelated phenomena.

\section{ACKNOWLEDGMENT}

This work was supported by the BMBF, Germany (Project Nos. 05 SB8 FKB and 05 SE8 FKA).
${ }^{1}$ N. W. Ashcroft and N. D. Mermin, Solid State Physics (Saunders College Publishing, Fort Worth, 1976).

${ }^{2}$ D. Schoenberg, Magnetic Oscillations in Metals (Cambridge University Press, Cambridge, 1984).

${ }^{3}$ Z.-X. Shen and D. S. Dessau, Phys. Rep. 253, 1 (1995).

${ }^{4}$ A. Santoni, L. J. Terminello, F. J. Himpsel, and T. Takahashi, Appl. Phys. A: Solids Surf. 52, 229 (1991).

${ }^{5}$ P. Aebi, J. Osterwalder, P. Schwaller, L. Schlapbach, M. Shimoda, T. Mochiku, and K. Kadowaki, Phys. Rev. Lett. 72, 2757 (1994)

${ }^{6}$ Y.-D. Chuang, A. D. Gromko, D. S. Dessau, Y. Aiura, Y. Yamaguchi, K. Oka, A. J. Arko, J. Joyce, H. Eisaki, S. I. Uchida, K. Nakamura, and Y. Ando, Phys. Rev. Lett. 83, 3717 (1999).

${ }^{7}$ H. M. Fretwell, A. Kaminski, J. Mesot, J. C. Campuzano, M. R. Norman, M. Randeria, T. Sato, R. Gatt, T. Takahashi, and K. Kadowaki, Phys. Rev. Lett. 84, 4449 (2000).

${ }^{8}$ S. V. Borisenko, M. S. Golden, S. Legner, T. Pichler, C. Dürr, M. Knupfer, J. Fink, G. Yang, S. Abell, and H. Berger, Phys. Rev. Lett. 84, 4453 (2000).

${ }^{9}$ L. Kipp, K. Rossnagel, C. Solterbeck, T. Strasser, W. Schattke, and M. Skibowski, Phys. Rev. Lett. 83, 5551 (1999).

${ }^{10}$ R. Claessen, R. Anderson, J. Allen, C. Olson, C. Janowitz, W. Ellis, S. Harm, M. Kalning, R. Manzke, and M. Skibowski, Phys. Rev. Lett. 69, 808 (1992).

${ }^{11}$ S. Harm, R. Dürig, R. Manzke, M. Skibowski, R. Claessen, and J. W. Allen, J. Electron Spectrosc. Relat. Phenom. 68, 111 (1994).

${ }^{12}$ R. Claessen, R. O. Anderson, G.-H. Gweon, J. W. Allen, W. P. Ellis, C. Janowitz, C. G. Olson, Z. X. Shen, V. Eyert, M. Skibowski, K. Friemelt, E. Bucher, and S. Hüfner, Phys. Rev. B 54, 2453 (1996).

${ }^{13}$ The data for the low-temperature $(30 \mathrm{~K})$ gold Fermi edge were numerically fitted under the assumption of a linearly increasing density of states, which is cut off by the Fermi-Dirac distribution and convoluted with a Gaussian spectrometer response function. With an energy resolution of $30 \mathrm{meV}$ FWHM the determination of the position of the Fermi energy is reliable within $\pm 1 \mathrm{meV}$.

${ }^{14}$ P. Hohenberg and W. Kohn, Phys. Rev. 136, B864 (1964).

${ }^{15}$ G. B. Bachelet, D. R. Hamann, and M. Schlüter, Phys. Rev. B 26, 4199 (1982).

${ }^{16}$ D. M. Ceperley and B. J. Alder, Phys. Rev. Lett. 45, 566 (1980).

${ }^{17}$ J. P. Perdew and A. Zunger, Phys. Rev. B 23, 5048 (1981).
${ }^{18}$ Y. Arnaud and M. Chevreton, J. Solid State Chem. 39, 230 (1981).

${ }^{19}$ Due to the threefold symmetry of the space group $P \overline{3} m 1\left(D_{3 d}^{3}\right)$ one distinguishes between the $\Gamma M A L$ and $\Gamma M^{\prime} A L^{\prime}$ planes. In the $\Gamma M^{\prime}$ direction the Te atom is situated above the Ti atom within the unit cell.

${ }^{20}$ J. C. Campuzano, H. Ding, M. R. Norman, M. Randeria, A. F. Bellman, T. Yokoya, T. Takahashi, H. Katayama-Yoshida, T. Mochiku, and K. Kadowaki, Phys. Rev. B 53, R14 737 (1996).

${ }^{21}$ Th. Straub, R. Claessen, P. Steiner, S. Hüfner, V. Eyert, K. Friemelt, and E. Bucher, Phys. Rev. B 55, 13473 (1997).

${ }^{22}$ M. C. Schabel, C.-H. Park, A. Matsuura, Z.-X. Shen, D. A. Bonn, R. Liang, and W. N. Hardy, Phys. Rev. B 57, 6107 (1998).

${ }^{23}$ P. J. Feibelman and D. E. Eastman, Phys. Rev. B 10, 4932 (1974).

${ }^{24}$ M. Randeria, H. Ding, J.-C. Campuzano, A. Bellmann, G. Jennings, T. Yokoya, T. Takahashi, H. Katayama-Yoshida, T. Mochiku, and K. Kadowaki, Phys. Rev. Lett. 74, 4951 (1995).

${ }^{25}$ P. Nozières, Theory of Interacting Fermi Systems (Benjamin, New York, 1964).

${ }^{26}$ K. Matho, Physica B 199\&200, 382 (1994).

${ }^{27}$ K. Matho, J. Phys. Chem. Solids 156, 1735 (1995).

${ }^{28}$ K. Matho, Mol. Phys. Rep. 17, 141 (1997).

${ }^{29}$ J. W. Allen, G.-H. Gweon, R. Claessen, and K. Matho, J. Phys. Chem. Solids 56, 1849 (1995).

${ }^{30}$ That the photoelectron contribution to the peak widths is negligible follows from a careful estimate of the band velocities perpendicular to the surface (Ref. 12).

${ }^{31}$ Note that $\epsilon_{\mathbf{k}}=\epsilon_{\mathbf{k}}^{0}+\Sigma(\mathbf{k}, 0)$, where $\epsilon_{\mathbf{k}}^{0}$ is the bare excitation energy and $\Sigma(\mathbf{k}, \omega)$ corresponds to the complex self-energy function. $\Sigma(\mathbf{k}, 0)$ is real and the FS condition is $\epsilon_{\mathbf{k}}=0$ (Ref. 28).

${ }^{32}$ The symmetry of the analyzer resolution function can best be checked by photoemission from rare gases using a gas cell.

${ }^{33}$ J. M. Luttinger, Phys. Rev. 121, 942 (1961).

${ }^{34}$ C. Hodges, H. Smith, and J. W. Wilkins, Phys. Rev. B 4, 302 (1971).

${ }^{35}$ C. M. Varma, P. B. Littlewood, S. Schmitt-Rink, E. Abrahams, and A. E. Ruckenstein, Phys. Rev. Lett. 63, 1996 (1989).

${ }^{36}$ K. Matho, Acta Phys. Pol. A 97, 1 (2000).

${ }^{37}$ M. R. Norman, H. Ding, M. Randeria, J. C. Campuzano, T. Yokoya, T. Takeuchi, T. Takahashi, T. Mochiku, K. Kadowaki, 
P. Guptasarma, and D. G. Hinks, Nature (London) 392, 157 (1998).

${ }^{38}$ P. Aebi, J. Osterwalder, R. Fasel, D. Naumovic, and L. Schlapbach, Surf. Sci. 307-309, 917 (1994).

${ }^{39}$ J. A. Con Foo, A. P. J. Stampfl, A. Ziegler, B. Mattern, M. Hollering, R. Denecke, L. Ley, J. D. Riley, and R. C. G. Leckey, Phys. Rev. B 53, 9649 (1996).

${ }^{40}$ The second value is taken from Ref. 9 where the $\Delta T$ method was used to determine the surface-parallel Fermi vector at $h \nu$
$=21.22 \mathrm{eV}$.

${ }^{41}$ N. V. Smith, Comments Condens. Matter Phys. 15, 263 (1992).

${ }^{42}$ J. A. Knapp, F. J. Himpsel, and D. E. Eastman, Phys. Rev. B 19, 4952 (1979).

${ }^{43}$ N. V. Smith, P. Thiry, and Y. Petroff, Phys. Rev. B 47, 15476 (1993).

${ }^{44}$ G. Ertl and J. Küppers, Low Energy Electrons and Surface Chemistry (VCH, Weinheim, 1985). 